\title{
MODELING OF ERRORS IN THE MEASUREMENT CHANNELS OF THE AUTOMATED PROCESS CONTROL SYSTEM IN THE LABVIEW ENVIRONMENT
}

\begin{abstract}
Аннотация. Исследованы вопросы оценки погрешностей измерительных каналов АСУТП. Предложен метод исследования погрешностей измерительных каналов, на основе их математического моделирования в среде визуального программирования LabVIEW.

Ключевые слова: измерительные каналы АСУTП, среда программирования LabVIEW, оценка погрешностей, моделирование измерительных каналов.

Abstract. Questions of estimation of errors of measuring channels of automated process control systems are investigated. A method for studying measurement channel errors based on their mathematical modeling in the visual programming environment LabVIEW is proposed.

Keywords: measurement channels of automated control systems, LabVIEW programming environment, error estimation, modeling of measurement channels.
\end{abstract}

Автоматизированные системы управления технологическими процессами (АСУТП) широко используются в химической и нефтехимической промышленности и других отраслях с непрерывным технологическим циклом.

Одной из основных подсистем АСУТП является измерительная система. Поэтому среди ряда требований, которые предъявляются к АСУТП как сложной технической системе, важное место занимают требования к метрологическому обеспечению АСУТП.

Основными структурными единицами измерительных систем являются измерительные каналы. Измерительные системы с их измерительными каналами, в соответствии с [1], «...обладают основными признаками средств измерений и являются их разновидностью», и, следовательно, на них распространяются метрологические нормы и правила. Однако измерительные каналы АСУТП рассматриваются как средства измерения особого рода [2] и относятся к новому типу средств измерительно-информационной техники - процессорных измерительных средств, принципиальная особенность которых заключается в том, что в них программируемая вычислительная мощность входит в состав измерительной цепи и участвует в получении результатов измерений.

При разработке систем автоматизации в техническом задании устанавливаются пределы допустимых погрешностей измерительных каналов основных технологических параметров. Перед проектировщиком стоит задача выбо- 
ра первичных преобразователей и других технических и программных средств, входящих в измерительный канал, суммарная погрешность которых не превышает установленных в техническом задании требований.

Расчет погрешностей измерительных каналов представляет собой трудоемкую задачу. Поэтому разработка программного обеспечения, позволяющего решить эту проблему, является актуальной задачей.

Для моделирования измерительных каналов АСУТП можно использовать среду визуального программирования LabVIEW (Laboratory Virtual Instrument Engineering Workbench), которая представляет собой среду графического программирования, предназначенную для создания прикладного программного обеспечения информационно-измерительных систем, а также различных компьютерных систем сбора и обработки экспериментальных данных.

Основной концепцией среды LabVIEW является виртуальный прибор (система организуется в виде программной модели реально существующего или гипотетического прибора). B среде LabVIEW можно создать новый виртуальный прибор с необходимыми данными, а можно использовать уже готовый. Разработчику виртуального прибора предоставляется графическая оболочка, включающая в себя весь набор инструментов, необходимых для сбора данных, их анализа и представления полученных результатов.

Исходя из вышеизложенного сорормулированы следующие задачи работы:

- провести анализ погрешностей всех элементов, входящих в измерительный канал: первичных преобразователей, нормирующих преобразователей, модулей ввода аналоговых сигналов контроллера, программного обеспечения;

- разработать модели погрешностей всех элементов, входящих в измерительный канал, в среде LabVIEW;

- провести анализ влияния погрешностей каждой из составляющих, входящих в измерительные каналы, на результирующую погрешность;

- на основании влияния погрешностей каждой из составляющих сфрормулировать рекомендации по конфигурированию измерительных каналов.

\section{ЛИТЕРАТУРА}

1. ГОСТ 34.003-90. Информационная технология. Комплекс стандартов на автоматизированные системы. Автоматизированные системы. Термины и определения. URL: http://docs.cntd.ru/document/gost-34-003-90.

2. ГОСТ 23222-88. Характеристики точности выполнения предписанной функции средств автоматизации. Требования к нормированию. Общие методы контроля.URL: http://docs.cntd.ru/document/gost-23222-88. 